\title{
The Internet of Things (IoT) and its Application Domains
}

\author{
Yusuf Perwej \\ Assistant Professor \\ Dept. of Information \\ Technology \\ Al Baha University, Al \\ Baha \\ Kingdom of Saudi Arabia
}

\author{
Kashiful Haq \\ Associate Professor \\ Dept. of Computer \\ Science \\ Meerut, India
}

\author{
Firoj Parwej \\ Assistant Professor \\ Dept.of Computer \\ Science \& Engineering \\ Delhi, India
}

\author{
Mumdouh M. \\ Mohamed Hassan \\ Assistant Professor \\ Dept. of Computer \\ Science \\ Al Baha University, Al \\ Baha \\ Kingdom of Saudi Arabia
}

\begin{abstract}
There is no suspicion that IoT has added a new dimension to the living being by the link between smart objects. Thus making the link among any media and anything at any place and anytime was appreciable. Under the umbrella of the Internet of Things (IoT) the number of interconnected devices is expected to grow exponentially toward more than 34 billion devices until 2021. IoT will propose the unique identification of the objects and their virtual representation as the basis for autonomously development of applications and services. These will be characterized by enormous and self-governing data capture, incident transfer, network connectivity and interoperability. This technology has a lot of applications in heterogeneous fields. The IoT technology and applications are likely to be major drivers of investment and innovation in the communications sector, over the forthcoming years, delivering the valued advantage to citizens, client and industrial end-users. These will lead to the introduction of many new and modern services. It will permit data to be transmitted between many various types of devices, enhance the safety of transportation, and decrease the consumption of energy and enhance our health. In this paper, we are briefly discussing about the Internet of Things and applications in several fields. The IoT applications are using at the edge of the network sensors accumulate data on a computing and communicating device and actuators to perform distinguished tasks controlled by these devices.
\end{abstract}

\section{Keywords}

IoT Smart Cities, Industrial Internet of Things (IIoT), IoT Health Care, IoT Smart Homes, IoT Platform, Energy Internet of Things (EIoT).

\section{INTRODUCTION}

The Big data movement was enabled by the amalgamation of technology drivers increasing volumes of unstructured data, low cost commodity servers and a thirst for faster answers [1], likewise a number of various technological advances have converged to enable the Internet of everything. The connecting every moment things embedded with electronics, software, and sensors to internet enabling to collect and exchange data without human interaction called as the Internet of Things (IoT) [2]. The Internet of Things is bridging the virtual world with the real world and the mobile networks need to scale to match the demands of 22-48 billion things while the processing capabilities need addressing the information provided by the digital shadow of these real things. Any technology available today has not reached to its $100 \%$ capability and it always has a limitation to go. So, we can say that the Internet of Things has a notable technology in a world that can help other technologies to reach its accurate and complete $100 \%$ capability as well. The IoT is the network of physic all objects that contain embedded technology to communicate and sense or communicate with their [3] internal states or the external environment. The confluence of efficient wireless protocols, cheaper processors, enhance sensors, and a bevy of startups and established companies developing the necessary management and application software, has finally made the concept of the IoT mainstream. Foremost, the smart home, connected appliances are what people think of when they hear IoT. They imagine an intelligent house, programmed to save energy and make your life a more comfortable one. The heating systems will be synced with external temperature sensors, alarm clocks will be synced with traffic apps, which will be synced with cost evaluations, lighting will respond as we enter an apartment, as might our coffee makers. It all sounds so alien to us, but there are plenty such homes that have already happened.In addition, IoT will have a drastic effect on waste management. Accompanied by the seamless integration of light, heat and air conditioning that reacts to you, a lot of money could be saved on those bills. We have our daily commute. Think of you left your home without your house key your house would tell you this. The cars will anticipate our viewpoint and open themselves via a sensor in our phones. Intelligent traffic detection will permit our device to direct us to the shortest route to work and home. The cab services for business meetings will be planned according to your calendar synced with your smartphone. In sport, IoT devices and wearables will be all about performance, efficiency [4]. You'll be able to track your progress, errors, power, agility, overall cariovascular fitness level and any variable you can imagine. Whether for cycling, tennis, or football, all can make use of sensors and they will use them to their full effect. The development of enabling technologies such as smart phones, embedded systems, cloud networking, network virtualization, Nano-electronics, communications, sensors, and software will be essential to provide to things the [5] capability to be connected all the time everywhere. This will also support important future IoT product innovations affecting many various industrial sectors. The main aim of IoT are the creation of smart environments and spaces and self-aware things for energy, mobility, climate, food, digital society and health applications. The time to come IoT developments will address highly distributed IoT applications involving a high degree of distribution, and processing at the edge of the network by using platforms that that provide compute, storage, and networking services between edge devices and computing data centers. The big data analytics tools have the capacity to handle huge volumes of data [6] generated from IoT devices that create a continuous stream of information. 


\section{INTERNET OF THINGS PLATFORM}

IoT platform is an essential component of a large IoT ecosystem that supports and connects all components within the system show in figure 1. An IoT platform is a multi-layer technology that enables uncomplicated provisioning, management, and automation of connected devices within the Internet of Things universe. It principally connects your hardware, however diverse, to the cloud by using resilient connectivity options, enterprise-grade security mechanisms, and broad data processing powers. The IoT platform confers a set of ready-to-use features that very much speed up development of applications for connected devices as well as take care of scalability and [7] cross-device compatibility. It is generally referred to as middleware when we talk about how it connects remote devices to user applications and handle all the interactions between the hardware and the application layers. IoT platforms originated in the form of IoT middleware, which main aim was to function as a mediator between the application layers and hardware. Its main action contains data collection of the devices over various protocols and network topologies, device management, remote device configuration and control, and over-the-air firmware improved. In real world miscellaneous IoT ecosystems, IoT middleware is expected to support concord with almost any connected device and combination in with third-party [8] applications used by the device. This freedom from the underlying hardware and overhanging software permits a single IoT platform to handle any kind of connected device in the same effortless way.

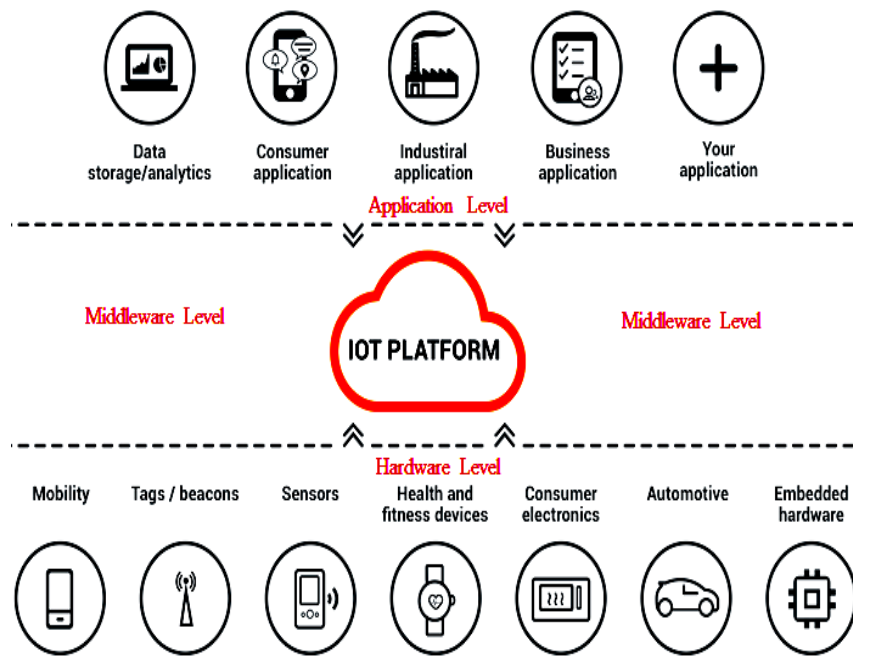

Fig 1. The Internet of Things Platform

\section{INDUSTRIAL INTERNET OF THINGS (IIOT)}

In this section, we briefly describe the overview of the IoT The industrial internet of things (IIoT) refers to the extension and use of the internet of things (IoT) in industrial sectors [9] and applications. A powerful emphasis on big data, machineto-machine communication, and machine learning, the IIoT enables industries and enterprises to have better efficiency and credibility in their operations. The Industrial Internet of Things is made up of a multitude of devices connected by communications software show in figure 2. The outcomes systems, and even the individual devices that comprise it, can exchange, analyze, monitor, collect, and instantly act on information to intelligently transform their behavior or their environment all without human intervention. The IIoT can be distinguished as a huge number of connected [10] industrial systems that are communicating and coordinating their data analytics and actions to enhance industrial performance and advantage society as a whole. The industrial systems that interface the digital world to the actual world via sensors and actuators that solve sophisticated control issues are commonly known as cyber-physical systems.

There are two points of view on how the Industrial IoT differs from the IoT. The first point of view is that there are two clearly separate areas of interest. The Industrial IoT connects critical machines and sensors in high-stakes industries like as transportation, healthcare, energy, and industrial control. These are systems in which lack of success often outcome in life-threatening or other emergency circumstances. IoT systems tend to be consumer-level devices such [2] as smart home thermometers, wearable fitness tools, and automatic pet feeders. They are important and appropriate, but breakdowns do not immediately create emergency circumstances. The second point of view sees the Industrial IoT as the infrastructure that must be built before IoT applications can be developed. The IoT, to some limitation, depends on the Industrial IoT.

The IIoT focuses unhesitatingly on intelligent cyber-physical systems. These systems consist of machines connected to computers that interpret, analyze and make decisions almost instantaneously, based on sensor data from many widely distributed sources. The IIoT enables the smart system in your car that brakes automatically when it detects an impediment in the road. It enables the sick person [2] monitoring system in hospitals to track everything from a patient's heart rate to their medication penetration. It enables a mining machine or space robot to shield and efficiently operate where humans can't [5]. The huge amounts of data are generated and transmitted between devices, system sensors and actual time systems. The actual time doesn't just mean fast, it means as fast as the machines can react, even if there are thousands of devices in a system, and hundreds of thousands of messages traveling via the system. The connectivity software, easy has to work and work rapidly, no matter how many devices are involved, and cooperation secures confidential system information along the way.

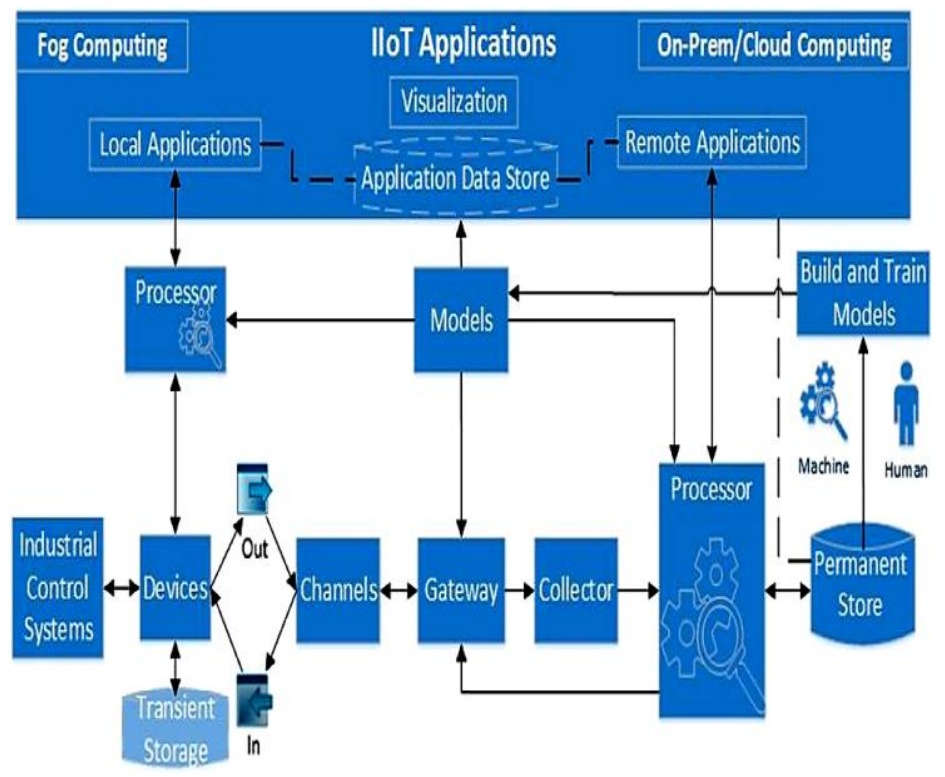

Fig 2. The Industrial Internet of Things (IIoT) Architecture 
The expected evolution of the Industrial IoT market will facilitate the invention of creative business models, it will be go with by the development of [11] new and the adoption of existing IoT technologies in more and more fields of application, and in the end enable the digital networking of the whole value chain across various domains.

\section{INTERNET OF THINGS IN SMART CITIES DOMAIN}

At present, roughly 73 percent of the world's population is expected to live in cities by 2050 according to Gartner [12]. This intense urban growth is already placing a considerable strain on the existing infrastructure, and with more people making the move to urban living, it's only going to get worse in the coming years. The IoT is everywhere nowadays, from industrial applications to emergency services, $\mathrm{p}$ city lighting and other smart city applications, public transportation, public safety, show in figure 3 . The IoT technologies implemented in [13] urban environments enable the creation of so-called smart cities and make better budget efficiency, the quality of life and the investment appeal of any city. The primary areas of application for IoT solutions in urban environments are public usefulness, transportation and services for residents. Besides, the numbers of IoT components and devices in 2017 and 2018 are expected to an increase of $44 \%$ and $46 \%$, respectively. By 2018, about 3.6 billion IoT components and devices are expected to be utilized in smart cities [12]. The smart city mission is to make better the old urban infrastructure in cities. The concept of smart cities is to make its existence in the development of urban development strategy. There are lot of existing technologies in urban cities, like as internet, wireless communication, infrared, Bluetooth [14], Wi-Fi differing from technologies and their range. Smart city is to make best use of public resources by increasing quality of services and decrease the expense.

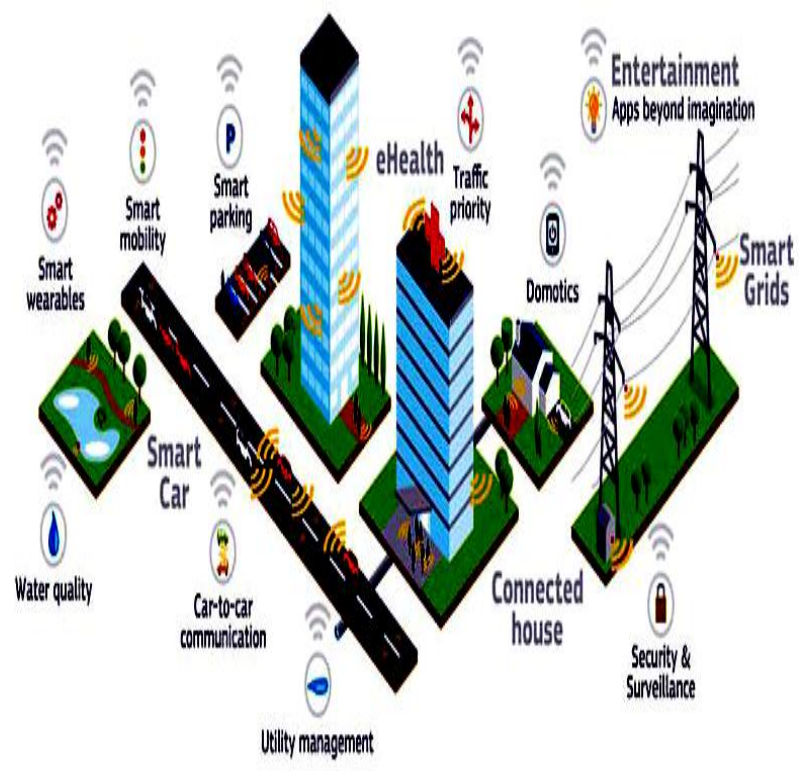

Fig 3. The Internet of Things Smart Cities

The primary aim of IoT in urban cities is to provide easy and unique access to public resources, so that preferable utilization and optimization of transport surveillance, power and maintenance [15] of public areas will be achieved. New technological solutions are needed to handle the increasingly scarce infrastructure resources, especially in view of the challenges enforce by population growth, typically limited financial resources. The IoT technologies and principles hold the promise of being able to ameliorate the resource management of many assets respectively to town life, including the flow of goods, the movement of private and public conveyance, and the greening of the environment. In smart cities applications, the IoT has been imagined as supporting a huge population of relatively low-bandwidth sensing devices, especially in M2M environments [16], and generally in stationary locations for example data collecting meteorological weather stations, electric meters etc.

\subsection{Internet of Things Use Cases for Smart Ciies}

In this section, we are overview some of the services that might be enabled by an urban IoT model and that are of potential interest in the smart city context, because they can realize the win-win circumstance of increasing the quality and ameliorate the services offered to the citizens while bringing an inexpensive advantage for the city administration in terms of reduction of the operational costs. The smart cities could be considered a collection of industries that contains waste water management, [17] city lighting, city transit, emergency services, traffic management and more. Actually, new IoT smart city projects are likely to emerge as the available technologies become more widely adopted, and more targeted at the need of specific use cases. For the state body to successfully increase efficiency, improve their services, and make better the quality of life for their residents, they necessity a platform that can power IoT applications for smart cities. The right one will combined, process, and interpret the data that smart devices [18] generate, make sure that the infrastructure is in place to take these cities into the new epoch of connectivity. Below, we are discussing an overview of the most popular use cases that are already implemented in smart cities across the globe.

\subsubsection{Effective Water Supply}

The IoT has the potential to transform the way town consume water. The smart meters can make better leak detection and data integrity and stop losing revenue due to inefficiency, and boost productivity [18] by lack of the amount of time spent entering and analyzing data. In addition, these meters can be designed to feature consumer facing portals, providing residents with real time access to information about their consumption and water supply.

\subsubsection{City Lighting}

The lighting is one of the most popular examples of IoT applications for smart cities, and many municipalities at present are turning to wireless communications for cost savings and energy deficiency. In this context, Lumca Lighting of Quebec City is putting smart city lighting to work [19]. 


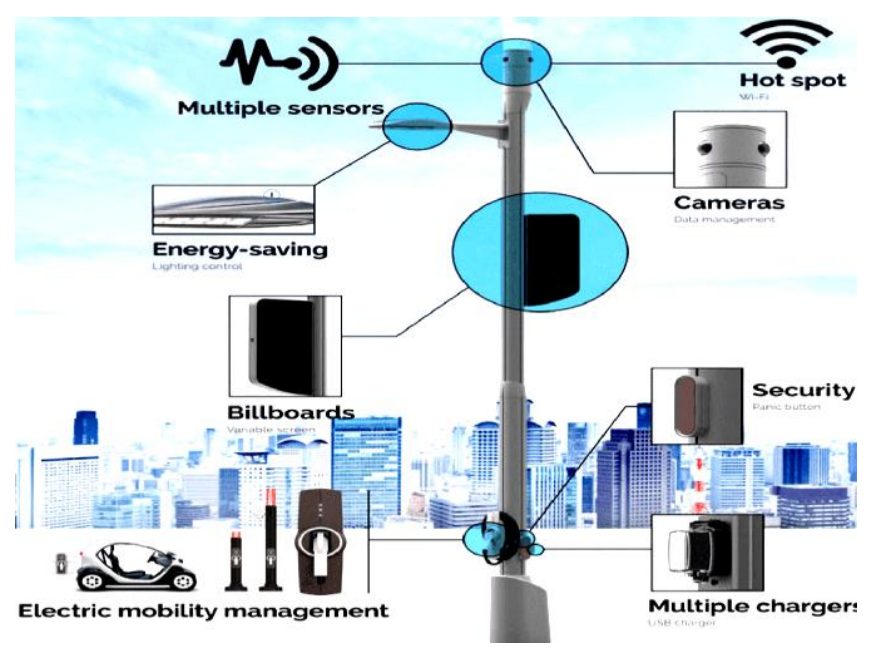

Fig 4. The Lumca Lighting Smart Pole

For instance, this outdoor lighting outfit developed an extensive lighting solution the lumca smart pole with built-in power, connectivity, sensors, and other intelligent characteristics, all of which are configured and handle through digi remote manager, a centralized software platform with a graphical user interface show in figure 4 .

\subsubsection{IoT in Road Traffic}

The smart cities make sure that their citizens move from point A to point B as safely and efficiently as possible. To attain this, municipalities turn to IoT development and execution smart traffic solutions. The smart traffic solutions use dissimilar types of sensors, bring in GPS data from drivers smart phones to control the number, location and the speed of vehicles. Concurrently, smart traffic lights connected to a [20] cloud management platform permit monitoring green light timings and automatically change the lights based on current traffic circumstance to put a stop to congestion. Besides, using historical data, smart solutions for traffic management can forecast where the traffic could go and take measures to put a stop to potential congestion.

\subsubsection{Citizens Safety}

For improved public safety, IoT based smart city technologies proposed real time monitoring, analytics, and decision-making tools. Integrate data from acoustic [21] sensors and CCTV cameras deployed ubiquitously the city with the data from a social media feed and analyzing it, public safety solutions can prophecy potential crime scenes. This will permit the police to prevent potential criminal.

\subsubsection{Garbage Management}

Most garbage collection operators empty containers according to predefined schedules. This is not a very efficient procedure since it leads to the unproductive use of garbage containers and unneeded fuel consumption by garbage collecting trucks. IoT-enabled smart city solutions help to optimize garbage collection schedules by tracking waste levels, also providing route optimization and operational analytics. Each garbage container gets a sensor that gathers the data about the level of the garbage in a container [22]. It is close to a certain threshold, the garbage management solution receives a sensor record, processes it, and sends a notification to a truck driver's mobile app. Consequently, the truck driver empties a full container, avoiding emptying half-full ones.

\subsubsection{Air Standard}

The European Union officially adopted a 20-20-20 renewable energy directive setting climate change reduction target for the next decade [23]. The aim to 20 percent reduction in greenhouse gas emissions by 2020 differentiate with 1990 levels, a 20 percent cut in energy consumption via make better energy efficiency by 2020 and a 20 percent increase in the use of renewable energy by 2020 . To such limit, an urban IoT can provide a means to monitor the standard of the air in crowded areas, parks or fitness trails [24]. Therewith, communication facilities can be provided to let health applications running on joggers' devices be connected to the infrastructure. The human being can always find the healthiest path for outdoor activities and can be continuously connected to their preferred particular training application. The realization of such a service needs that air standard and pollution sensors be deployed across the city and that the sensor data to be made publicly available to townsman.

\subsubsection{Smart Parking}

The assist of GPS data from drivers' smartphones, smart parking solutions determine whether the parking spots are engaged or not engaged and create a real-time parking map. When the nearest parking spot becomes free, drivers receive a notification and use the map on their phone to discover a parking spot faster and effortless as an alternative of blindly driving around.

\subsubsection{Crowdsensing}

The crowdsensing allows a huge population of mobile devices to measure incident of common interest over an extended geographic area, enabling the big data collection, [25] sharing, and analysis. It has major urban applications for the active or passive collection of traffic conditions, weather conditions, and even video images. The crowdsensing leverages everywhere mobile devices and the increasingly more pervasive wireless network infrastructure to collect and analyze sensed data without the necessity to deploy a huge set of stable sensors.

\subsubsection{Energy Efficient Buildings}

The IoT technology is making it effortless for buildings with legacy infrastructure to save energy and make better their sustainability. The smart building energy management systems, for example, [26] cooling, lighting, use IoT devices to connect disparate, nonstandard heating, and fire-safety systems to a central management application. Research shows that commercial buildings, waste up to 35 percent of the energy they use, so savings with a smart building energy management system can be valuable.

\subsubsection{Trustworthy Public Transportation}

The public transportation is disrupted whenever there are road closures, nasty weather, or equipment breakdowns etc. IoT can give transit authorities the real-time [27] understanding they need to implement contingency plans, and make sure that residents always have access to secure, trustworthy, and efficient public transportation. This might be done using insights from cameras or connected devices at bus shelters or other public territory.

\subsubsection{Environment}

The IoT driven smart city solutions permit tracking parameters critical for a healthy environment in order to maintain them at a good level. For instance, to supervise water quality, a city can deploy a network of sensors across the water grid and connect them to a cloud management [24] platform. Sensors measure $\mathrm{pH}$ level, the amount of dissolved 
oxygen and dissolved ions. If leakage happens and the chemical composition of water varies, the cloud platform triggers an output defined by the users. For example, if a Nitrate (NO3-) level exceeds $1 \mathrm{mg} / \mathrm{L}$, a water quality management solution warning maintenance teams of fouling and automatically creates a case for field workers, who then start fixing the difficult circumstances.

\section{INTERNET OF THINGS IN ENERGY DOMAIN}

The Internet of Things (IoT) has already started to make a clear influence in the energy sector. From sensors to monitor the temperature in a room to sophisticated applications that control the energy use of a whole building, IoT technology in the energy sector is cutting costs and creating more productive, connected buildings [28]. The power industry searches for a new model of distribution and management that can incorporate renewables into the grid, the use of the Internet of Things (IoT) devices can provide solutions by flexibly managing demand. The Energy Internet of Things (EIoT) can decrease the cited challenges by coordinating electric demand with electric supply, this can speed up the adoption [29] of renewable and sustainable electricity technologies. The Internet of Things plays an important role in the field of energy management and regulation.

But renewable energy comes with a drawback. In view of the fact that, renewable energy frequently relies on environmental inputs, it inherits the environment's natural mutable. This mutable causes problem in energy distribution when the energy generated by a solar or wind farm is especially high or low. Too much energy being put into the grid consequence of wasted energy, too little energy consequence of the need to periodically power up more stable fossil fuel power sources. These practices of powering up fossil fuel plants or wasting energy are presently the optimal solutions, but the introduction of IoT enabled technologies could present a more inimitable system.

This is where twenty-first-century technology such as the Internet of Things (IoT), Big Data [1], and Machine Learning come into the picture. They assist harness clean power along with optimization and can facilitate optimal utilization of resources. For instance the IoT and connected appliances can aid in the smooth running of devices that are energy intensive, so that they operate efficiently and alienate unexpected increases in power demand. In this context, individual building sensors can cut energy use dramatically by simply monitoring [30] the lighting and temperature when the building is not being used. Fire sensors that warning people to the nearest exit and also count the amount of people exiting at each location can make better safety in buildings as well. Energy Internet of Things (EIoT) in the home has produced devices such as programmable thermostats that handle electricity use while homeowners are unavailable. IoT provides a distinguished way for maintaining and monitoring a low cost and high level of care. It explores energy problems in the same way as functional problem in a complex business network and provides preferable solutions.

\subsection{Energy Internet of Things Utilization}

In an EIoT scenario, demand would be improved to match the supply produced by renewable and traditional energy resources. Internet connected appliances would use sensors to monitor and change consumer energy use. EIoT devices could measure moment-to-moment variability in electricity generation and adjust their operations correspondingly [31]. The EIoT devices can be used to enable the integration of renewable energy generation into the electric grid and renewable generation mutable could be directly measured by EIoT devices on the grid, sensors on the grid, or a central control. These EIoT devices could then respond swiftly by increasing, lowering electricity loads, reduce the negative effects of renewable generation mutable on the grid. Any excess or loss between the renewable power output and prediction can be swiftly utilized by EIoT devices. EIoT devices would receive communications that renewable output was not being similar prediction and modify their operations suitably. EIoT devices provide a distinctive, readily available, and rapid responding resource that can provide transformation in load that respond swiftly to transformation in supply.

\subsection{Energy Internet of Things Component}

At credible of EIoT ecosystem is the best innovation to facilitate them sustainable energy grid of the future. A primary component of the EIoT ecosystem is the EIoT element or device. An EIoT device is a device that controls, energy storage, consumption, production, while having all the feature of an IoT device, like as internet connectivity [32]. EIoT devices with information storage, connectivity, sensors, smart controls, processing power, and cloud management creates the capability to find a solution to the intermittency, mutable, and unpredictability currently limiting the potential of renewable energy generation. The utilization of renewable energy sources and innovation in EIoT can alter historic patterns of generation and consumption. This creates new patterns of electricity production and usage that dissimilar to the ways in which electric grid operators manage systems at present. EIoT devices will need outside data to optimize their operations. These data form datasets, based on information from reliable weather prophecy services, electric grid cost and loads, and other data applicable to control of EIoT devices.

\subsection{Network Component in Energy Internet of Things}

Another primary component of the EIoT ecosystem is the network. In order for EIoT to be triumphantly implemented, electronic devices must be able to easily connect to the utility's extranet, to the cloud, and to the Internet. There are three plans conceived for managing EIoT coordination firstly a centrally planned and controlled energy management platform, secondly a web of independent control devices, making their own decisions about optimizing, thirdly a compromise between the two that utilizes gateway devices [33]. The all EIoT devices would communicate via the internet with one central energy management platform, likely hosted in the cloud. This platform would take in device operation schedules, price signals, sensor measurements, and renewable energy generation levels. In the secondly each smart EIoT device communicates over the Internet directly with other EIoT devices. The EIoT devices from two dissimilar homes and one business communicate with each other, not a central control, to optimize their operations. A cloud-based data source provides third-party data like weather and electricity prices. The management of grid irregularities are mediating between EIoT devices as they collect grid data and communicate with each other about the best energy use for each device. In the thirdly the gateways arrangement the smaller local hubs, or gateways, can act as localized central control devices and manage EIoT devices in that building. These gateways optimize EIoT operations, without the complexity of a full web control strategy. This arrangement decreases the need for each EIoT device to handle multiple connections and negotiations. The gateway can house high power CPUs and extensive memory, and optimize each 
building's EIoT operation, while the mediate best plan with other buildings. This plan decreases communications volume, decrease EIoT complexity, but limits flexibility as each EIoT device depends on the gateway for operations scheduling.

\subsection{Internet of Things Use Cases in Energy}

The IoT system provides a strong means of managing the consumption cost of energy and optimize the output of enterprises. Energy management is becoming vital for both utilities and facilities with the ever growing demand for energy supply. In this context, the evolution of the IoT is revolutionizing energy management systems suitable for energy utilization [32]. IoT is considered to play a pivotal role in creating these energy management systems smarter in the forthcoming year. The Internet of Things is enabling energy efficiencies while [28] creating a new way for energy conservation. This will make you efficient in energy utilization while keeping the costs to a minimum.

\subsubsection{Smart Meters}

Conventionally, an electricity meter would log the amount of electricity you use in your home, after which an employee from your electricity provider reads it and bills you accordingly. An analog meter needs an employee to read it, while a smart meter transmits your energy consumption to the company for you to be billed analog meters have no way of letting you know they've lost power should an emergency [33] arise, smart meters, on the other hand, send a notification to the company to alert them over power outages. Analog meter needs yearly servicing to make sure accuracy, and smart meters only need a battery replacement every 8-20 years.

\subsubsection{Mending and Maintenance}

The remotely monitored, real-time sensors can detect any lack of success or unusual decrease in energy efficiency. These findings can be accessed instantaneously from any Internet connected device and users can be alerted to unusually by SMS message or email.

\subsubsection{Collect Consumption Data Easy}

An end of month, money spent on manual on-site meter readings and data processing of water, gas and electricity consumption. One time activated, connected meters instantly start transmitting data over the Sigfox public network with no pairing or configuration need, and run for years without replacing the battery. You can now monitor and optimize your infrastructure in real time to detect leaks and breakdowns, and service providers can automate billing and remotely operative and deactivate services.

\subsubsection{Monitor Water Services and Remotely}

IoT sensors provide crucial data on essential water infrastructure conditions, which assistance impedes malfunctions and flooding as well as improving maintenance crew efficiency. It's never been so easy to remotely measure water levels, $\mathrm{pH}$, salinity, ORP, flow, pressure, turbidity to impede overflows, track drinking water supply and monitor the waste water network and treatment operations.

\subsubsection{Electricity Pole Surveillance}

The tilting of electricity poles as an outcome of strong wind, traffic accidents, earth movements can reason mechanical tension and cable breakage. To impede the pole falling completely, it is necessary to locate the trouble swiftly and carry out remedial action. Periodic measurements performed remotely by IoT devices enable deterrent maintenance, and alerts are sent if major tilting occurs. In this context, maintenance crews can now act before the pole falls and identify where urgent repairs are needed.

\section{INTERNET OF THINGS IN HEALTH CARE DOMAIN}

A new paradigm, known as the Internet of Things (IoT), has a comprehensive applicability in many areas, including health care. The full application of this paradigm in healthcare area is a mutual hope because it permits medical centers to function more competently and patients to obtain superior treatment show in figure 5. Technology can assist overcome limitations [34] in resources, financial and personnel, can reach patients living long distances from health care specialists, and can assist communities deal with aging populations. The use of this technology based healthcare procedure, there is unparalleled advantage which could make better the quality and efficiency of treatments and accordingly make better the health of the patients. The IoT to be more specific, Internet of Healthcare Things (IoHT), is revolutionizing the healthcare industry. The number of connected medical devices is expected to increase from 10.5 billion to 52 billion over the next decade. The ability to capture, store, analyze, and disseminate health information using technology can decrease the human resources needed to manage this process and make better the accuracy of the data. The ability to perform a quick analysis on this health data can make sure that scarce health care resources are allocated where needed most urgently. In remote, rural areas of many parts of the real world, health care providers and services are often not available locally, need patients travel long distances to obtain care. Technology, such as mobile device-based video conferencing, can permit patients to [35] be diagnosed and potentially treated remotely by doctors and other health care professionals.

IoT will become the biggest source of data on Earth. Just imagine the possibilities if laboratory test outcome, personal statistics, human-oriented data, like medical history, allergies to medication, amongst many other things, were to be digitized as part of the electronic health initiative. Healthcare practitioners will be able to interpret and leverage the multiplicity of big data from connected systems to make informed patient care decisions as well as understand and prophecy current and future health trends. The IoT expands the potential for using technology to support health care by connecting not only people, applications, and data, but also sensors and devices that collect biometric and contextual data. The IoT based systems can be make use of to address a broad set of health problem ranging from well-being to sickness, physical to mental health, preventive care to treatment or rehabilitation, and temporary disabilities to chronic disease. In this scenario, smartphones, watches, and other smart devices as well as additional sensors, devices, and equipment can be connected to IoT networks to provide information about the sick person, the sick person activities, and the sick person context or environment. 


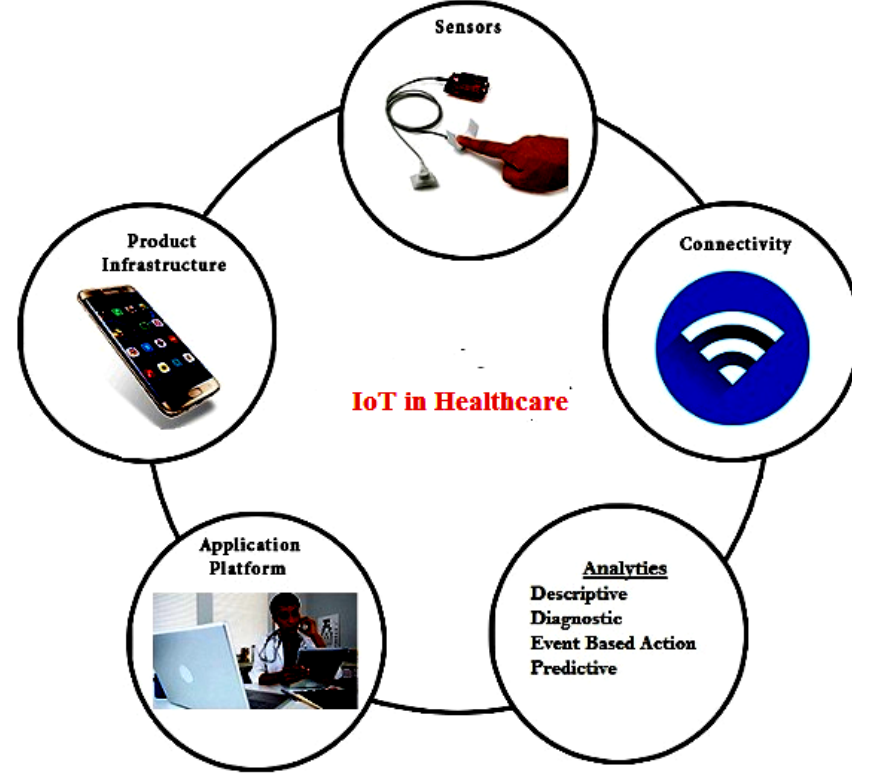

Fig 5. The Internet of Things Healthcare

\subsection{The Healthcare Ecosystem}

The Internet of Healthcare Things, IoHT in short, is a concept that describes uniquely identifiable devices connected to the Internet and able to interact with each other, used in the medical domain. An ecosystem to represent the complicated and interconnected nature of the relationships between the human participants, the autonomous and subordinate devices, [36] and the applications, interfaces, and data. This type of system or ecosystem is dissimilar from the early e-health systems that enabled a sick person health care data to be stored digitally and accessed remotely via the Internet and mobile devices, or the ability to have an online consultation with a physician. Presently via the IoT, connected devices also store, access, and share data with other components of the ecosystem.

\subsection{Internet of Things Use Cases in Healthcare}

The rise of the Internet of Things (IoT) is resulting in some exciting advancements in the 21 st century. The Internet of Things is normally defined as a network of day-to-day devices, appliances, and other objects equipped with computer chips and sensors that can collect and transmit data via the Internet. In an environment where healthcare remains notably expensive to the average citizen, the overall age of the global population is increasing, and the prevalence of chronic diseases is on the rise, the advent of the Internet of Things shows assurance in helping healthcare facilities operate more efficiently. This technology is set to change the healthcare industry within the next decade, as it has great potential and multiple potential applications, from remote monitoring to medical device integration [37].

\subsubsection{At the Same Time Monitoring and Reporting}

The real time monitoring via connected devices can save lives in the event of a medical emergency like asthma attacks, heart failure, diabetes, etc. The real-time monitoring of the condition in place by means of a smart medical device connected to a smartphone app, connected devices can collect medical and other needed health data and use the data connection of the smartphone to transfer collected information to a physician. The IoT device collects and transfers health data, such as blood pressure, oxygen, blood sugar levels, weight, and ECGs. These data are stored in the cloud and can be shared with an authorized person, who could be a physician, your insurance company, a participating health firm or an external consultant, to permit them to look at the collected data regardless of their place, time, or device.

\subsubsection{End-to-end Affordability and Connectivity} IoT can automate sick person care workflow with the assistance, healthcare mobility solution and other new technologies, and next generation healthcare facilities. IoT enables interoperability, machine-to-machine communication, information exchange, and data movement that makes healthcare service delivery effective [38]. The connectivity protocols like as Bluetooth LE, Wi-Fi, Z-wave, ZigBee, and other modern protocols, healthcare personnel can transform the way they spot illness and sickness in sick persons and can also innovate revolutionary ways of medication.

\subsubsection{Data Classification and Analysis}

The large amount of data that a healthcare device sends in a very short time owing to their real time application is hard to store and handle if the access to the cloud is unavailable. Even for healthcare providers to obtain data originating from multiple devices and sources and analyze it manually is a tough prerequisite. IoT devices can collect, report and analyses the data in actual time and cut the need to store the raw data. This all can come about overcloud with the providers only getting access to final reports with graphs.

\subsubsection{Tracking and Warning}

IoT permits devices to gather important data and transfer that data to doctors for real-time tracking, while dropping notifications to people about critical parts via mobile apps and other linked devices [38]. IoT enables actual time alert, tracking, and monitoring, which permits hands-on treatments, better accuracy, appropriate intervention by doctors and ameliorate complete sick person care delivery outcome.

\subsubsection{Remote Medical Assistance}

In the event of an emergency, the sick person can contact a doctor who is many kilometers away with a smart mobile apps. Using mobility solutions in healthcare, the medics can instantaneously check the sick person and identify the illness on-the-go.

\subsubsection{Lower Costs}

The utilize of IoT solutions and connected medical device assent healthcare providers to monitor sick persons in real time. This means less unneeded visits to doctors, and fewer hospital stays and readmissions thanks to an efficient data collection and management.

\subsubsection{Better Sick Person Experience}

To connect the health care system via the Internet of things, patients gets more engaged in their treatment, and doctors, make better diagnostic accuracy since they have all the necessary sick person data at hand.

\subsubsection{Preferable Management of Drugs and Medicine}

The IoT provides solutions that assent for better management of drugs as well as ensuring a preferable understanding of and adherence to instructions by the sick person. Interconnected machines sharing data permit hospital [35] staff to spend less time searching for drug information, tracking regulations, and inventorying supplies. The IoT monitoring solutions have demonstrated that they can assist sick persons adhere to their 
treatment schedules while allowing doctors to track compliance with prescriptions.

\subsubsection{Minimize Errors and Waste}

The utilize of IoT data collection and workflow automation is an excellent way to cut down on waste such as unnecessary tests and expensive imaging, minimize system costs and minimize errors.

\subsubsection{Improved Outcomes of Treatment}

The healthcare solutions that are connected via cloud computing and use big data, can provide caregivers with the ability to access real time data which can utilize to make informed decisions and to provide evidence-based treatments.

\section{INTERNET OF THINGS IN SMART HOMES DOMAIN}

The Internet of Things has become a worldwide recognized term in workplaces and homes, and in a literal sense could be used to describe anything that is connected to the internet show in figure 6 . The IoT is the next step in the evolution of wireless networks, Big data, and connected devices [39], as sensors shrink in size and migrate from our smartphones to other day-to-day objects. One of the greatest eventuality still lies ahead in the form of the smart home [40]. A smart, connected home is an instance of this paradigm, inheriting all the aspects of connectivity of the devices involved. A smart, connected home is a residence equipped with sensors, systems, and devices that can [41] be remotely accessed, controlled, and monitored, typically via the Internet. This can include anything from cell phones, smart bulbs, fitness trackers, smart speakers and dishwashers, all the way to water quality sensors in pumping stations. The chance for interconnection are endless. Smart home automation is very famous due to its numerous advantage in a promising area, these techniques will controls all the electronic devices which will decrease the human involvement to get minimize. Home automation systems where a hub is used, it is common to find one or more of the Z-Wave, RedTacton [42], BidCoS and ZigBee, Li-Fi [43], communication protocols that provide low-latency data transfer and power consumption [40] lower than Wi-Fi. The hub, then connected via Wi-Fi or wired Ethernet, allowing connection from remote devices or cloud services. Smart home technologies can unlock both individual and society-wide advantage in dissimilar ways. They can provide economic saving, make better convenience for consumers, contribute to more ecological and sustainable living, reinforce the buyer's sense of safety and security, and more [44].

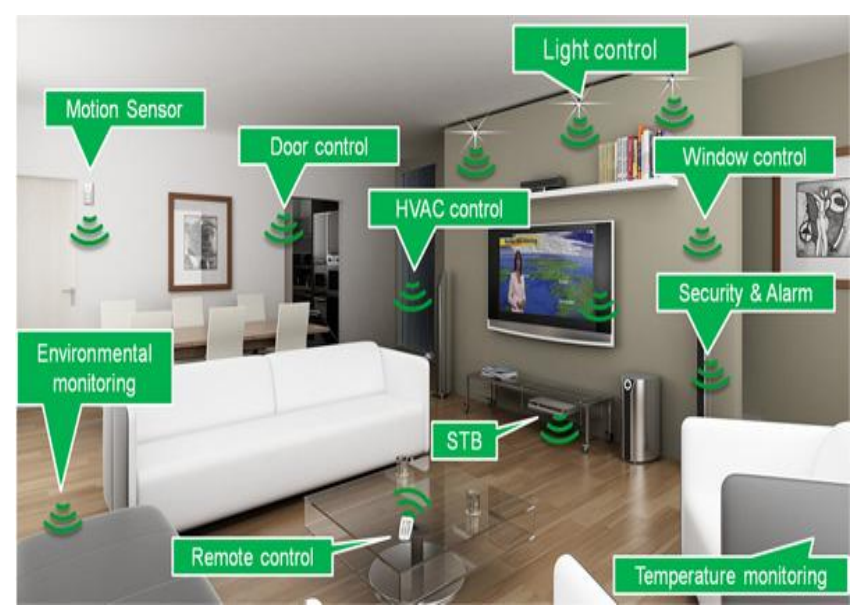

Fig 6. The Internet of Things SmartHome

\subsection{The Smart Home Stakeholders}

Smart homes need melange control in its different gadgets which are basically electronic appliances. This has revolutionized the area of home automation with respect to a fast increased level of affordability and clarity via the integration of home appliances with smart phone and tablet connectivity. In IoT applications, [39] such as smart homes, there are diverse stakeholders ranging from [45] technology investors, technology developers, technology integrators show in figure 7. The specific stakeholders may be involved depending on the real smart home system.

\subsubsection{Device Manufacturers}

The appliance manufacturers, including smart product suppliers like as smart meter and entertainment device manufacturers. Householders [46] may purchase devices directly from manufacturers, but oftentimes via retailers or service providers. Instance of manufacturers company are Honeywell, Samsung, and LG.

\subsubsection{End Users}

The end user is the stakeholder that uses the services. Usually, this represents the home residents that purchase and operate the different smart, connected home devices and services etc.

\subsubsection{Network Providers}

The telecommunication providers supply and handle the network infrastructure like core network, radio access network, and interconnectivity, network to service providers that want to proposal smart home services. Effectively they are the stakeholders that connect the householders to the Internet. For instance, Verizon.

\subsubsection{Regulators}

The regulators are outer entities overseeing business services or specific industry sectors. This can include certification and accreditation bodies belonging to quality, security, and protection. An instance of this could be a privacy regulator that develops laws for protection personally identifiable information from inappropriate exploitation. Presumably, this entity may affect the entire spectrum of industry stakeholders.

\subsubsection{Platform Providers}

Entities supplying mechanisms, tools, and frameworks help in overcoming integration challenges and facilitating convenient assess, customization, and automation support. Generally, various device manufacturers work with various platform providers for third-party integration. Some organizations serving this role are Apple, Google, and Amazon.

\subsubsection{Service Providers}

The application service providers and utility companies that provide the end users with hardware equipment to support or enable various smart connected home services. Three examples of service providers are AT\&T, verisure, and Leak Defense. 


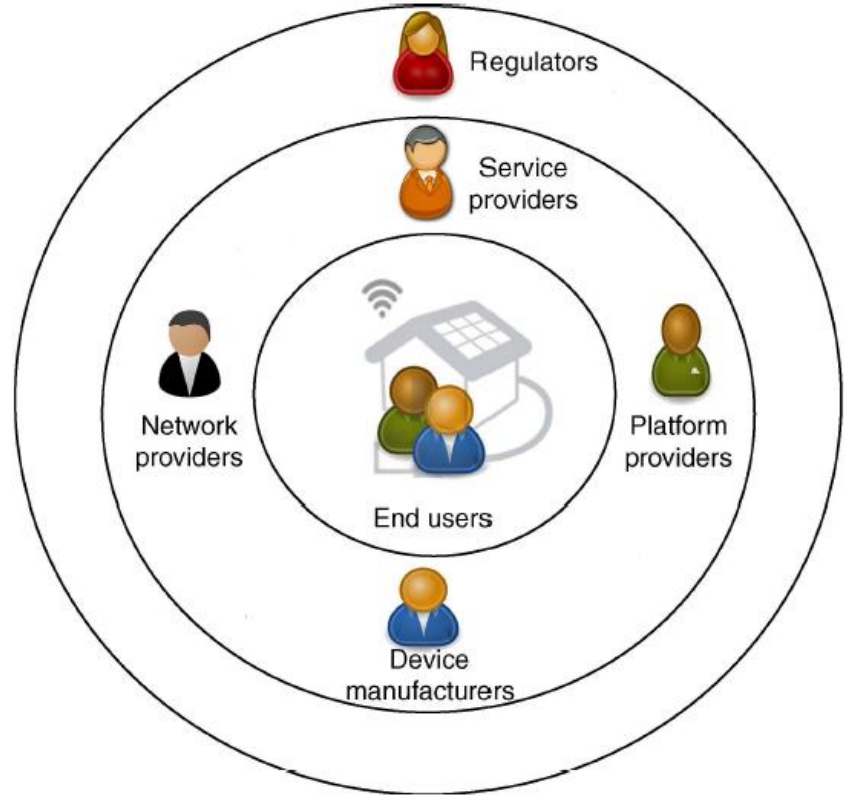

Fig 7. The Smarthome Stakeholders

\subsection{The Smart Home Architectures}

The architecture of a computing system defines the types of system components together with their interaction patterns. The architecture of smarthome is strongly impacted by the computational capabilities of their components. There are two main architectural styles of smart, connected homes first centralized and second distributed.

\subsubsection{Centralized Architecture}

In a centralized smarthome architecture, the control system is realized by means of a computer system that is accountable for data acquisition from sensors, user interfacing, as well as with the implementation of control algorithms and sending instructions to the actuators. In this model, all the data are retrieved by a single central entity. This entity is frequently a dedicated local gateway that houses the application logic, stores data, and communicates with the IoT devices that are connected to it [47]. Besides to the control function, it is accountable for interfacing the smarthome with the outside world via Internet, as well as by providing services to the home residents. An option is to limit the capacity of the home gateway to data obtaining, software interfacing with domotic devices and primary process in [48].

\subsubsection{Distributed Architecture}

In a distributed smarthome architecture, the software of the control system is conceptualized and implemented as a distributed computing system. The distributed architecture advantage from the computational resources of smart objects to embed software components into the nodes of the smarthome network. Addition, the smarthome architecture can be only conceptually distributed, while still physically centralized into the home gateway. In differentiating to the centralized approach where information flows via a common node, in the distributed model, the architecture is same as to that of a peer-to-peer system and information is only exchanged when needed. It can be argued that connected devices in this model are considered smart on their own. Distributed architectures can use a service-oriented approach or a mobile agent approach [49]. The main drawback of distributed model is that applying network security is more complicated when compared to the centralized approach.

\subsection{Internet of Things Use Cases for Smart Home}

The smart connected home can integrate various smart home services to provide a convenient, advantageous, and safe environment to the household members [50], as well as to assist them perform their household tasks effectively. In general, smart connected home systems can be organized into four categories as systems that support health care, security services, energy, and entertainment.

\subsubsection{Health Care}

The health care service area is concentrated on providing mobile health care and fitness support, and target to provide independent healthy living. In differentiating to the other domains, the health care service also involves the use of wearable sensors in order to permit for possibly continuous monitoring of body signal parameters even while not at home. This contains connected devices like as physiological devices, fitness monitors, and wireless scales.

\subsubsection{Security Services}

The systems in the security category are often targeted for proposal services, which are designed to monitor, find out, and control security and safety threats. Smart home security and safety systems typically range from remote entrance monitoring services for systems that automatically [45] recognize physical threats, namely a fire or a burglary, and autonomously take the corresponding action. This domain contains functionality that support alarm systems, cameras, and smart door locks.

\subsubsection{Energy}

Energy systems are aimed to provide efficient energy consumption and management for the home. The energy domain commonly involves the use of smart meters, smart thermostats, and adaptive lighting systems. System architectures in this domain may utilize intelligent multiagent systems and control strategies to prophecy and automatically maximize energy efficiency and user convenience.

\subsubsection{Entertainment}

The smart connected home systems tend to promote entertainment typically maximizing occupants' comfort and facility by providing personalized [45] amusement content and social communication services. The entertainment sector commonly involves smart speaker systems, connected TVs, and game consoles.

\section{INTERNET OF THINGS IN AGRICULTURE DOMAIN}

In IoT based smart farming, a system is built for monitoring the crop field with the assist of sensors like as light, humidity, temperature, soil moisture, and automating the irrigation system. The farmers can monitor the field conditions from anyplace. The applications of IoT based [51] smart farming not only aim traditional, huge farming operations, but could also be new levers to uplift other growing or common trends in agricultural like organic farming, family farming and improve highly transparent farming. IoT-based smart farming can provide great advantage, including more efficient water usage, or optimization of inputs and treatments [53]. IoT recent trends in agriculture are using technology in order to make smarter decisions, decrease costs, and boost production. 


\subsection{Internet of Things Agriculture Architecture}

The IoT technologies can play important roles in the agriculture implementation. IoT provides not only the communication infrastructure to interconnect every smart object from sensors, vehicle, to the user mobile device via the Internet, but also functions including local and remote data obtaining, in-cloud intelligent information, analysis and decision-making, data access, visualization, user interfacing, and agriculture [53] operation automation show in figure 8 . IoT has two points of view, to be either smart device centric or Internet centric. The agriculture IoT model has three fundamental layers. The bottom is the data, obtaining layer, in which environmental and crop data are collected via either sensors or remote sensing devices like as UAV then uploaded to the cloud storage via an Internet gateway. The second layer is the cloud computing function layer.

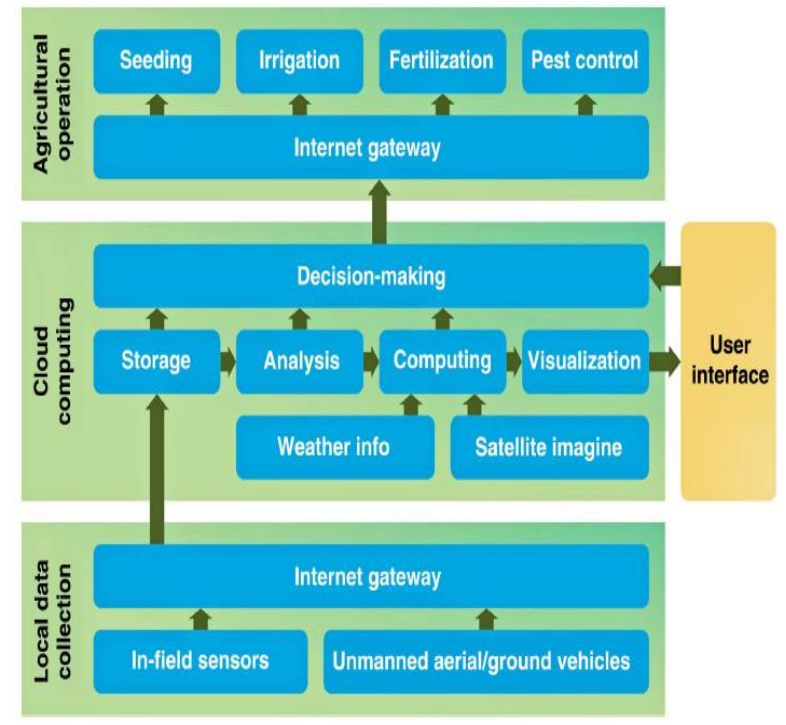

Fig 8. IoT Architecture in Agriculture

The miscellaneous cloud computing functions is services are integrated and provided in this layer [52]. For any need function, there are always various solutions available from various providers. In the general process, first of all raw data will be filtered and processed by data analysis tools to abstract information. Subsequently, this authenticity information will be converted to necessary knowledge of data mining and machine learning tools. The consideration of specific factors, like as soil condition, fertilizing pattern, crop state, weather and environment, circumstances and so forth, knowledge derived [51] in the last step will be used for decision-making or the generation of field index maps for various intentions. This layer also provides data visualization and presentation services for user access. The top layer is the control of all agricultural operations, including fertilizing, seeding, irrigation, and harvesting etc.

\subsection{Internet of Things Use Cases for Agriculture}

Internet of Things networks of objects that interact with other objects via internet holds potential to transform today's agriculture by enabling more appropriate resources management via low-cost sensors and generating huge amounts of data for effective decision-making. Farming has seen a number of technological alterations in the last decades, becoming more industrialized and technology driven. In this section, we are exploring the IoT use cases in agriculture sector.

\subsubsection{Monitoring of Climate Conditions}

Perhaps the most famous smart agriculture gadgets are weather stations, integrate different smart farming sensors. Located across the field, they collect different data from the environment and send it to the cloud. The provided measurements can be used to map the climate conditions, select the suitable crops, and take the required measures to make better their capacity.

\subsubsection{Autonomous Tractors}

Autonomous tractors aren't just mindless driving robots. They also operate with intelligence to maximize farming dexterity. Robotics collaborated to make tractors that use path generating algorithms calculate the most efficient area coverage pattern in a field taking into account the type of action, vehicle, size of implementing, number of vehicles in the field, implement turn radius.

\subsubsection{Precision Farming}

The precision farming can be idea of as anything that makes the farming implementation more controlled and well-aimed when it comes to raising livestock and growing of crops. In this method of farm management, a key component is the use of IT and different items like robotics, autonomous vehicles, sensors, control systems, automated hardware, variable rate technology, and so on. The precision agriculture is one of the most renowned applications of IoT in the agricultural sector and numerous organizations is leveraging this technique around the world.

\subsubsection{Greenhouse Automation}

In addition to sourcing, environmental data, weather stations can automatically coordinate the conditions to match the given parameters. In particular, greenhouse automation systems use an almost identical principle. For example, Farmapp and Growlink are also IoT agriculture product offering like as capabilities among others.

\subsubsection{Agricultural Drones}

At present, agriculture is one of the major industries to incorporate drones. Drones are being used in agriculture in order to increase different agricultural practices. The ways ground-based and aerial based drones are being used in agriculture are crop monitoring, crop spraying, crop health assessment, irrigation, planting, and soil and field analysis etc. The major advantage of using drones include crop health imaging, integrated GIS mapping, effortless to use, saves time, and the potential rise yields.

\subsubsection{Crop Management}

Using of IoT product in agriculture and another element of precision farming are crop management devices. Similar to weather stations, they should be placed in the field to collect data specific to crop farming from temperature and precipitation to leaf water potential and overall crop health.

\subsubsection{Livestock Monitoring}

Huge farm owners can utilize wireless IoT applications to collect data regarding the location, well-being, and health of their cattle. This information, assist them in identifying animals that are sick so they can be separated from the herd, thereby stop the spread of disease. It also lowers labor costs as ranchers can locate their cattle with the assistance of IoT based sensors. 


\subsubsection{End-to-End Farm Management Systems}

A more complicated approach to IoT products in agriculture can be represented by the so-called farm productivity management systems. They normally include a number of agriculture IoT devices and sensors, installed on the premises as well as a powerful dashboard with analytical capabilities and in built accounting and reporting characteristics. This proposed remote farm monitoring capabilities and permit you to streamline most of the business operations.

\section{OTHER APPLICATIONS DOMAIN IN INTERNET OF THINGS (IoT)}

The Internet of Things (IoT) is a concept that involves connecting endpoint devices and physical objects to the Internet [2]. These objects can communicate with other objects to know each other's status and share information and data. IoT applications domain assurance to bring large value into our lives. In this section, we are exploring the other application domain IoT

\subsection{IoT Biometrics Domain}

IoT plays an important role in the biometrics security system such as a eye scanner system, fingerprint system, voice recognition system, etc. Presently, a biometric system is something that we always encounter in our everyday life. We every time either use a fingerprint sensor or an eye scanning system, depends on company to company. The conventional methods such as login and password for authentication on a smart node does not in fact compliment the IoT technology When objective is to achieve efficiency with IoT, conventional authentication methods become a bottleneck to it. In this context, passwords can be forgotten, guessed or shared, compromising security and exposing the endpoint [54]. It can also compromise the security of other IoT devices if similar password is used. The combining password by an additional factor of authentication to achieve two factor authentication ameliorate security, but removes convenience and user experience as well. All these shortcomings of conventional authentication methods can be fixed by using biometrics for IoT endpoint authentication. Biometrics makes use of an individual's physiological and behavioral characteristics like iris patterns, vein pattern, fingerprint patterns, to identify the user. Since these features are unique to an individual, they can be used as a secure procedure for authentication.

\subsection{IoT Transportation Domain}

IoT plays a vital role in all the fields of transportation as water-transportation, air-transportation, and land transportation. All the component of these transportation fields is built with smart devices such as sensors, processors and interconnected via cloud server or various servers that transmit data to networks. IoT in transportation is not only for making a journey from one place to another, but it also makes safer and more suitable. For instance, [55] a smart car performs work at the same time like as communication, entertainment, navigation, efficient, more reliable travel. IoT facilitates travelers to abide seamlessly connected to every means of travel. The vehicle is connected with the miscellaneousness of wireless standards to the internet such as Bluetooth, Wi-Fi, 3G, 4G, intelligent traffic system, and even to other vehicles. IoT in the transportation has come up with an advanced form of geo-fencing. It captures the location of an asset or device with the coordinates of a specific area. Geofencing assistance in starting the automated tasks. IoT in the transportation industry is the most gain from geo-fencing. It allows you to receive a warning when a driver wanders from the prescribed path, as it can bring delay in delivery time and cause an accidental loss.

\subsection{IoT Businesses Domain}

In the business, IoT not only means to connect the devices to the Internet, in spite of that, it is more than that. Presently, IoT is transforming the business enterprises by creating the eventuality to get smarter about the product, services, and the client experience. IoT is playing an eventuality to both clients as well as a service provider by understanding the client behavior and their needs. When product and service provider comprehend how its client use their product, they can better fulfill their needs and improve the client experience [56]. As IoT data offer the actual time operation, companies can respond swiftly to issues and request as they arise. IoT provides power of business to collect and analyze more data from different sources such as from their local setups and networks. This data lead more eventuality for automatic product updates or upgrades, tracing and tracking the assets. As the huge volume of data flow from data centers, production systems, sensors and IoT systems to the business enterprise at actual or non actual time assistance the enterprise to offer eventuality for innovation and development.

\section{CONCLUSION}

The term Things on the Internet of Things refers to anything and each thing in daily life which is accessed or connected via the internet. The IoT technology is cultivate and demonstrating the features in different applications need the integration of the highest, most generalized layer of intelligence and user interface that ties together link into devices and web services using interoperable platforms that deliver the functionality needed by the end-users. It inhabits in the ways the IoT is used to leverage the insights from automate, digitize, digitalize, data, optimize and in more fully developed stages transform processes, business models and even industries in a scope of digital mutation. The IoT is an advanced automation and analytics system which deals with sensor, networking, and artificial intelligence, cloud messaging, to deliver complete systems for the product or services. The whole working process of IoT starts with the device themselves, like as digital watches, electronic appliances, smartphones, which securely communicate with the IoT platform. The platforms collect and analyze the data from all various devices and platforms and shifting the most valuable data with applications to devices. In the end, this paper will give a better understanding for the new researchers in this field of IoT application domains.

\section{ACKNOWLEDGEMENTS}

The author would like to thank the reviewers anonymous for their constructive comments and that helped whit their revision to improve the resulting quality of this paper. I would like to express my gratitude towards my family and colleges for their co-operation and help me in completing this paper. 


\section{REFERENCES}

[1] Yusuf Perwej, "An Experiential Study of the Big Data," International Transaction of Electrical and Computer Engineers System (ITECES), USA, ISSN (Print): 23731273 ISSN (Online): 2373-1281, Vol. 4, No. 1, page 1425, March 2017, DOI:10.12691/iteces-4-1-3.

[2] Yusuf Perwej, Mahmoud A. AbouGhaly, Bedine Kerim, Hani Ali M. Harb. "An Extended Review on Internet of Things (IoT) and its Promising Applications" Communications on Applied Electronics (CAE), ISSN : 2394-4714, Foundation of Computer Science FCS, New York, USA, Volume 9, Number 26, PP 8- 22, February 2019, DOI: $10.5120 /$ cae2019652812

[3] Kim, J., Lee, J.W., 2014. OpenIoT: an open service framework for the Internet of Things. In: Proceedings of IEEE World Forum on Internet of Things (WF-IoT), pp. 89-93.

[4] Yusuf Perwej, Majzoob K. Omer, Osama E. Sheta, Hani Ali M. Harb, Mohmed S. Adrees, "The Future of Internet of Things (IoT) and Its Empowering Technology" International Journal of Engineering Science and Computing (IJESC), ISSN : 2250-1371, Volume 9, Issue No.3, Pages 20192- 20202, March 2019.

[5] Yusuf Perwej, Firoj Parwej, Mumdouh M. M. Hassan, Nikhat Akhtar, "The Internet-of-Things (IoT) Security: A Technological Perspective and Review", International Journal of Scientific Research in Computer Science, Engineering and Information Technology (IJSRCSEIT), ISSN : 2456-3307, Volume 5, Issue 1, Pages 462-482, February 2019, DOI: 10.32628/CSEIT195193

[6] Nikhat Akhtar, Firoj Parwej, Yusuf Perwej, "A Perusal Of Big Data Classification And Hadoop Technology," International Transaction of Electrical and Computer Engineers System (ITECES), USA, ISSN (Print): 23731273 ISSN (Online): 2373-1281, Vol. 4, No. 1, page 2638, May 2017, DOI: 10.12691/iteces-4-1-4.

[7] Oleksiy Mazhelis, Pasi Tyrväinen,"A framework for evaluating Internet-of-Things platforms: Application provider viewpoint", IEEE World Forum on Internet of Things (WF-IoT), IEEE, Seoul, South Korea, March 2014

[8] A. Abdelgawad, K. Yelamarthi, "Internet of Things (IoT) Platform for Structure Health Monitoring", Wireless Communications and Mobile Computing, vol. 2017, no. 6560797, pp. 10, 2017.

[9] L.D. Xu, W. He, S. Li, "Internet of things in industries: A survey", IEEE Transactions on Industrial Informatics, vol. 10, no. 4, pp. 2233-2243, Nov. 2014.

[10] M. Wollschlaeger, T. Sauter, J. Jasperneite, "The future of industrial communication: Automation networks in the era of the internet of things and Industry 4.0", IEEE Industrial Electronics Magazine, vol. 11, no. 1, pp. 1727, Mar. 2017.

[11] J. Wurm, K. Hoang, O. Arias, A.R. Sadeghi, Y. Jin, "Security analysis on consumer and industrial IoT devices", Proc. 21st Asia and South Pacific Design Automation Conference (ASP-DAC), pp. 519-524, Jan. 2016.

[12] Gartner. Gartner Says Smart CitiesWill Use 1.6 Billion Connected Things in 2016. 2015. Available online: https://www.gartner.com/newsroom/id/3175418, (accessed on 6 March 2019)

[13] Tomas Gea, Josep Paradells, Mariano Lamarca, David Roldán," Smart Cities as an Application of Internet of Things: Experiences and Lessons Learnt in Barcelona", Seventh International Conference on Innovative Mobile and Internet Services in Ubiquitous Computing, IEEE , Taichung, Taiwan, July 2013

[14] Yusuf Perwej, Kashiful Haq, Uruj Jaleel, Sharad Saxena, "Some drastic improvements found in the analysis of routing protocol for the Bluetooth technology using scatternet" International Conference on Computing, Communications and Information Technology Applications (CCITA-2010), Ubiquitous Computing and Communication Journal (UBICC) Seoul, South Korea, ISSN Online 1992-8424, ISSN Print 1994-4608, Volume CCITA-2010, Number 5, pages 86-95, 2010

[15] Albert Meijer and Manuel Rodriguez Bolivar "Governing the smart city: a review of the literature on smart urban governance", International Review of AdministrativeSciences, Vol 82, no. 2, pp. 392-408, 2016

[16] S. K. Datta, C. Bonnet, N. Nikaein, "An IoT gateway centric architecture to provide novel M2M services", Internet of Things (WF-loT) 2014 IEEE World Forum on, pp. 514-519, March 2014

[17] M. Dohler, I. Vilajosana, X. Vilajosana, and J. LLosa, "Smart Cities: AnAction Plan,"Barcelona Smart Cities Congress 2011, Barcelona, Spain,Dec. 2011.

[18] A. Zanella, N. Bui, A. Castellani, L. Vangelista, M. Zorzi, "Internet of things for smart cities", IEEE Internet of Things Journal, vol. 1, no. 1, pp. 22-32, Feb 2014

[19] N. Ouerhani, N. Pazos, M. Aeberli, J. Senn, S. Gobron, "Dynamic Street Light Management - Towards a citizen centered approach", 3rd Int. Conference on Hybrid City, 2015

[20] J. Jin, J. Gubbi, S. Marusic, M. Palaniswami, "An information framework for creating a smart city through internet of things", IEEE Internet of Things Journal, vol. 1, no. 2, pp. 112-121, April 2014.

[21] A. Zanella et al., "Internet of Things for Smart Cities", IEEE Internet of Things J., vol. 1, no. 1, pp. 22-32, 2014

[22] J. Jin et al., "An Information Framework for Creating a Smart City Through Internet of Things", IEEE Internet of Things J., vol. 1, no. 2, pp. 112-21, 2014

[23] Decision No 406/2009/Ec of the European Parliament and of theCouncil of 23 April 2009 on the effort of Member States to reducetheir greenhouse gas emissions to meet the Community's greenhousegas emission reduction commitments up to 2020

[24] A. R. Al-Ali, I. Zualkernan, and F. Aloul, "A mobile GPRS-sensorsarray for air pollution monitoring."'IEEE Sensors Journal, vol. 10, no.10, pp. 1666-1671, Oct. 2010.

[25] J. Liu, H. Shen, X. Zhang, "A survey of mobile crowdsensing techniques: A critical component for the Internet of Things", Proc. 25th Int. Conf. Comput. Commun. Netw. (ICCCN), pp. 1-6, Aug. 2016. 
[26] I. Ganchev, Z. Ji, M. O'Droma, "A Generic IoT Architecture for Smart Cities", 25th IET Irish Signals \& Systems Conf. 2014 and 2014 China-Ireland Int'l. Conf. Info. and Commun. Technologies, pp. 196-99, 2013.

[27] J. Gubbi et al., "Internet of Things (IoT): A Vision Architectural Elements and Future Directions", Future Generation Computer Systems, vol. 29, no. 7, pp. 164560, 2013.

[28] Jianli Pan, Raj Jain Subharthi Paul, Tam Vu, Abusayeed Saifullah, Mo Sha," An Internet of Things Framework for Smart Energy in Buildings: Designs, Prototype and Experiments",IEEE Internet of Things Journal, 2 (6) (2015), pp. 527-537

[29] Kabalci Ersan, Alper Gorgun, Yasin Kabalci, "Design and implementation of a renewable energy monitoring system", Power Engineering Energy and Electrical Drives (POWERENG) 2013 Fourth International Conference, 2013

[30] Andreas S. Spanias," Solar energy management as an Internet of Things (IoT) application", 8th International Conference on Information, Intelligence, Systems \& Applications (IISA), IEEE, Larnaca, Cyprus, Aug. 2017

[31] Ahmad A., Xiaoyun Z., Daji Q., Ahmed K.," An Energy-Efficient Relaying Scheme for Internet of Things Communications", IEEE International Conference on Communications (ICC), Kansas City, MO, USA, May 2018

[32] Conti, F., Schilling, R., and Schiavone, P.D. (2017) An IoT endpoint system-onchip for secure and energyefficient near-sensor analytics. IEEE Transactions on Circuits and Systems I: Regular Papers, 64(9) 24812494.

[33] Paek, J., Gnawali, O., Vieira, M., and Hao, S. "Embedded IoT systems: network, platform, and software. Mobile Information Systems" ,Article ID 5921523, 2017

[34] N. Zhu et al., "Bridging e-health and the Internet of Things: The SPHERE project", IEEE Intell. Syst., vol. 30, no. 4, pp. 39-46, Jul./Aug. 2015

[35] Y. Yin, Y. Zeng, X. Chen, Y. Fan, "The Internet of Things in healthcare: An overview", J. Ind. Inf. Integr., vol. 1, pp. 3-13, Mar. 2016

[36] C. A. Tokognon, B. Gao, G. Tian, Y. Yan, "Structural health monitoring framework based on Internet of Things: A survey", IEEE Internet Things J., vol. 4, no. 3, pp. 619-635, Jun. 2017.

[37] B. Xu, L. D. Xu, H. Cai, C. Xie, J. Hu, F. Bu, "Ubiquitous data accessing method in IoT-based information system for emergency medical services", IEEE Trans Ind. Informat., vol. 10, no. 2, pp. 1578-1586, May 2014.

[38] P. Gope, T. Hwang, "BSN-care: A secure IoT-based modern healthcare system using body sensor network", IEEE Sensors J., vol. 16, pp. 1368-1376, Mar. 2016.

[39] Twinkle Gondaliya, "A Survey on an Efficient IoT Based Smart Home", International Journal of Review in Electronics and Communication Engineering, vol. 4, no. 1, February 2016.
[40] Thomas Gonnot, Won-Jae Yi, Ehsan Monsef, Jafar Saniie, "Home Automation Device Protocol (HADP):A protocol Standard for Unified Device Interactions", Advances in Internet of Things, vol. 5, pp. 27-38, 2015.

[41] Balta-Ozkan, N., Davidson, R., Bicket, M., and Whitmarsh, L.,"Social barriers to the adoption of smart homes. Energy Policy”, 63, PP 363-374, 2013

[42] Yusuf Perwej, "A Literature Review of the Human Body as a Communication Medium using RedTacton" Communications on Applied Electronics (CAE), ISSN : 2394-4714, Foundation of Computer Science FCS, New York, USA, Volume 9, No.4, Pages 7 - 17, April 2016, DOI: $10.5120 /$ cae2016652161

[43] Yusuf Perwej, "The Next Generation of Wireless Communication Using Li-Fi (Light Fidelity) Technology," Journal of Computer Networks (JCN), USA, ISSN (Print): 2372-4749 ISSN (Online): 23724757, Science and Education Publishing Vol. 4, No. 1, page 20-29, June 2017. DOI:10.12691/jen-4-1-3

[44] Joao Lima, "Behold the 10 biggest IoT investments," Computer Business Review, April 9, 2015

[45] David Bregman, "Smart Home Intelligence - The eHome that Learns", International Journal of Smart Home, vol. 4, no. 4, pp. 35-46, October 2010.

[46] Boban Davidovic, Aleksandra Labus, "A smart home system based on sensor technology", Electronics and Energetics, vol. 29, no. 3, pp. 451-460, September 2016.

[47] Roman, R., Zhou, J., and Lopez, J. (2013) On the features and challenges of security and privacy in distributed internet of things. Computer Networks, 57(10), 2266-2279.

[48] M. Skubic, G. Alexander, M. Popescu, M. Rantz, andJ. Keller. A smart home application to eldercare:Current status and lessons learned.Technology andHealth Care, 17(3):183-201, Aug 2009.

[49] C.L. Wu, C.F. Liao, and L.C. Fu., "Serviceorientedsmart-home architecture based on osgi andmobile-agent technology", IEEE Transactions onSystems, Man, and Cybernetics, Part C: Applicationsand Reviews, 37(2):193-205, march 2007.

[50] Badica, C., Brezovan, M., and Badica, A.," An overview of smart home environments: architectures, technologies and applications", BCI (Local), 78, 2013

[51] Meonghun Lee, Sunchon Nat, Jeonghwan Hwang, "Agricultural Production System Based on IoT", Computational Science and Engineering (CSE) 2013 IEEE 16th International Conference, pp. 833-836, Dec 2013.

[52] Duan Yan-e, "Design of Intelligent Agriculture Management Information System Based on IoT", Intelligent Computation Technology and Automation (ICICTA) 2011 International Conference, vol. 1, pp. 1045-1049, March 2011

[53] Junyan Ma, Xingshe Zhou, Zhigang Li, "Connecting Agriculture to the Internet of Things through Sensor Networks", Internet of Things (iThings/CPSCom) 2011 International Conference on and 4th International Conference on Cyber Physical and Social Computing, pp. 184-187, Oct 2011. 
[54] Peter Corcoran, Claudia Costache, "Biometric technology and smartphones: A consideration of the practicalities of a broad adoption of biometrics and the likely impacts", Technology and Society (ISTAS) 2015 IEEE International Symposium on, pp. 1-7, 2015.

[55] Benjamin Yee Shing Li ; Lam Fat Yeung ; Kim Fung Tsang," Analysing traffic condition based on IoT technique", IEEE International Conference on Consumer Electronics - China, IEEE, Shenzhen, China, April 2014

[56] Zhuming Bi ; Li Da Xu ; Chengen Wang,” Internet of Things for Enterprise Systems of Modern Manufacturing ", IEEE Transactions on Industrial Informatics , Volume: 10 , Issue: 2 , PP 1537 - 1546, May 2014 